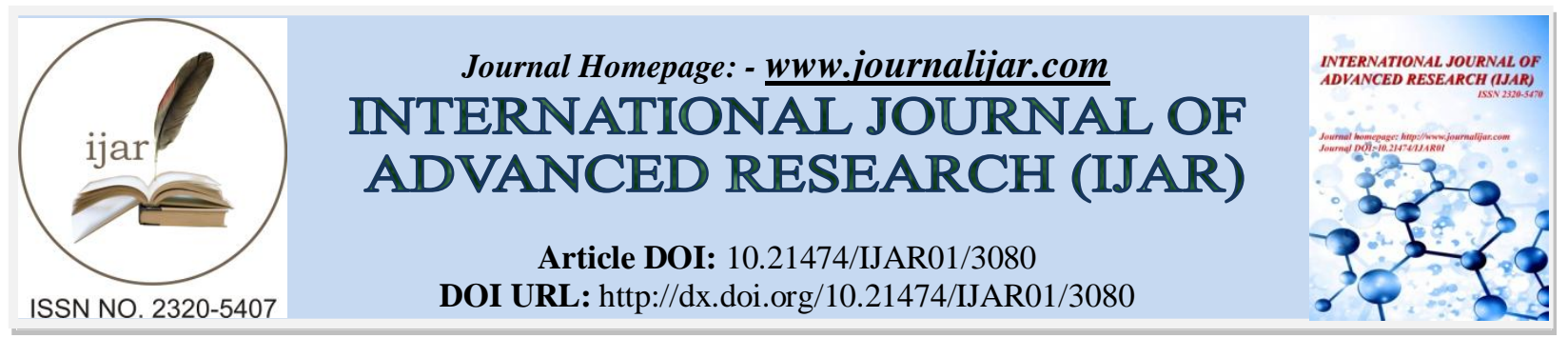

RESEARCH ARTICLE

\title{
MULTIPLE ACTIVITY BASED BIO-BOTANICAL WATER DISPERSIBLE GRANULES FORMULATION \& THEIR EFFICACY FOR PEST MANAGEMENT IN AGRICULTURE.
}

\author{
Smriti Kala ${ }^{1}$ Nisha Sogan $^{2}$ and P.K Patanjali ${ }^{1}$. \\ 1. Institute of Pesticide Formulation Technology, Gurgaon. \\ 2. National Institute of Malaria Research, New Delhi.
}

\section{Manuscript Info}

an......................

Manuscript History

Received: 03 November 2016

Final Accepted: 28 December 2016

Published: January 2017

Key words:-

WDG, Antifungal, \& Nanoparticles

\section{Abstract}

WDG formulation containing neem oil and metallic nanoparticles were prepared and evaluated for their efficacy against different pests nematode, fungus and cotton leaf worm. Various metallic nano particle 1;ike $\mathrm{Ag}$ and $\mathrm{Cu}$ alone and in combinations were tested for their efficacy against different pest. The process parameters and composition were optimized to get more improved formulation in terms of performance \& other quality parameters such as Suspensiblity, Dispersibility \& Particle size, which affects performance of formulation during its application. The prepared formulation was tested against different pests like fungus, nematodes and further for antifeedant activity. S3( mixture of $\mathrm{Cu}+\mathrm{Ag}$ ) provides highest $42 \%$ antifeedancy, $73 \%$ mortality in nematodes, and no fungal growth even after 48hrs. The present formulation S3 shows antifungal, nematicidal and antifeedant properties all in one activities in single formulation. The present S3 WDG formulations are safe and promising alternates to minimize the risk of dustiness, contamination.

Copy Right, IJAR, 2016,. All rights reserved.

\section{Introduction:-}

Pests have characteristics of damaging or harming agriculture through feeding on crops. Pests of different types either insects, fungus, nematodes infests variety of crops. These pests damage different parts of plant and are thus responsible for large loss in yield and also affect quality of crops. In order to protect crops from damage, pest management is a necessary tool. Different methods of pest control have been proposed by the investigators. For any pest control to be effective two important criteria are, improvement in quality of crop and crop protection.

To improve quality of crop, it is necessary to protect them from pests. Various synthetic pesticide formulations are used in agriculture against pests. But extensive use of these chemical pesticides has created environmental effects that are harmful to humans. These formulations are used for certain specific type of pest so user has to apply one or more different type of pesticide depending upon target pest. Because of such repetitive use of pesticide for different pests in crop protection, the problem associated with their use has become a major problem in recent years since there is issue of pesticide residue in most of the food commodities along with the soil. Pesticide residue accumulates in food, water and is toxic to human and environment (Lee et al; 2001). Harmful effects of synthetic pesticides are not limited to their toxicity alone, infact repetitive usage of pesticide has resulted in resistant pest, resistant pest are difficult to manage and required novel insecticide (Mecedo et al; 1997), which are bio botanical insecticide. 
Bio Botanical based pesticidal formulation can resolve above problem associated with synthetic pesticide since they are safe, biodegeredable and non persistent in the nature.

Accordingly there is need for pesticide composition containing no chemical pesticide and posses wide range of activity in terms of pest control, Which must be used against different pests. Neem oil commercially available is economical may be a safe alternative that can be used in place of synthetic pesticide. Neem itself has insecticidal, antifeedant (Shrivastava D.K. 2014) and also to some extent antifungal and nematicidal properties. The efficiency is not adequate, however neem oil has very superior antifeedant properties (Munetaka Ishida 1992). Whereas metallic nanopaticles

Having size range below 100nm are versatile agents with variety of properties (fungicidal, nematicidal ) (Sahar M. Ouda 2014) because of high surface area the best part is that metallic nanoparticles used as antifungal and nematicidal agent are required in very low concentrations. i.e they are equally effective when used in small amount.

Keeping above facts in consideration, We have made efforts to develop a bio-botanical based WDG pesticide formulation with multifunctional activities like fungicidal, nematicidal, antifeedant and which will also decrease the load of pesticide in the environment by decreasing the number of sprays, replacing synthetic pesticide by biopesticide and at the same time to improve crop health along with enhancements of crop yield.

Water-dispersible granules (WG) are pesticide formulations that are almost free from dust and easy to handle in packing when compared to liquid formulation. They are used in a manner similar to the known water-dispersible powders (WP) (H.J. Niessen 1983)The WDG formulations are safe and promising alternative to WP formulations which have problem of dustiness, contamination and dilution.

WG formulations of pesticides are produced by different processes like Pan Granulation, Fluid-bed Spray Granulation and Extrusion (Knowles, 2008). The WDG were prepared by Extrusion process are easy to prepare also the process is industrial feasible. The formulations developed have been evaluated at laboratory scale.

\section{Materials \& Method:-}

Materials:-

Neem oil, Silver Nitrate, Sodium borohydride and Lactose Monohydrate was obtained from Qualigens, Silica was obtained from Madhu silica pvt ltd, China Clay was obtained from IAC Minerals (Chennai, India), Wetting agent Sodium Naphthalene Sulphonate and Dispersing agent Lignin Sulphonate, Polycarboxylate were supplied by Albright \& Wilson Ltd. (Mumbai, India),

\section{Method:-}

Preparation of Metallic Nanoparticles:-

Metallic nano particles of silver and copper were prepared in laboratory by chemical reduction method using salts (Silver Nitrate and Copper sulphate pentahydrate) and reducing agent Sodium borohydride (Kandarp Mavani, 2013). PVP was used as capping agent for both salts. For copper naopartilces Ascorbic acids was used as an antioxidant (LIU Qing-ming 2012). Prepared nanoparticles were characterized by Malvern Zetasiser.

\section{Preparation of WDG containing Metallic Nanopaticles and neem oil:-}

WDG was prepared by Extrusion method (D. Ian Wilson 2007) using laboratory mini screw extruder (Model-Caleva Mini Screw) fitted with screen of $1 \mathrm{~mm}$ perforations. Neem oil and metallic nanoparticles were absorbed on silica; wetting and dispersing agents were added and mixed in mixer to get homogenous powder. Finally china clay was used as filler. Suitable amount of water was added to mixture and mixed properly with spatula .The final mixture was extruded in extruder to get granules. The extruded granules were dried in the oven. The composition of emulsion and WDG is given in Table 1 
Table 1:- Composition of WDG.

\begin{tabular}{|c|c|}
\hline Ingredients & \% w/w \\
\hline Neem oil & 20 \\
\hline $\begin{array}{c}\text { Metallic Nanoparticels } \\
\text { (S1, S2 \& S3) }\end{array}$ & 5 \\
\hline Silica & 30 \\
\hline Wetting Agent & $5-10$ \\
\hline Dispersing Agent & $8-10$ \\
\hline Lactose ( Dilutend) & 20 \\
\hline China Clay (Dilutend) & q.s. \\
\hline Total & 100 \\
\hline
\end{tabular}

Accodingly three samples were prepared S1, S2 and S3. Composition is same for all three samples only difference is $\mathbf{S 1}$ contains Silver Nanoparticles, $\mathbf{S 2}$ (Copper Nanoparticles) and $\mathbf{S 3}$ (Copper nanoparticle + Silver Nanoparticles).

\section{Suspensibility Analysis:-}

Suspensibility (suspension stability) is the percent part of active ingredient suspended in a column of water after a given time time period. Suspensibility of the WG formulations has significant role in the application of spray suspension. If the suspensibility is upto the mark, spray tank mixing requirement is less and homogeneity is maintained in the suspension. It ensures uniform distribution of active ingredient on the target area. The suspensibility of the samples was analyzed by method no. MT168 of CIPAC Handbook (CIPAC, 2007).

\section{Dispersibility Analysis:-}

Dispersibility is the percentage of active ingredient dispersed in water after stirring the suspension. The spray tank mixing requirement is minimum if the WG formulation has good dispersibility. The dispersibility is a major quality parameter of WG formulations. The dispersibility of the samples was analyzed by method no. MT174 of CIPAC Handbook (CIPAC, 2007).

\section{Particle Size Analysis:-}

The particle sizes of the samples were analyzed by particle size analyzer (Model- Malvern Master Sizer MS20) using distilled water as dispersant. The Suspensibility, Dispersibility \& particle size shown in Table 3

\section{Bioefficacy Experiments:-}

The formulation developed was tested for antifungal, Nematicidal and Antifeedant activity. The formulation show remarkable activity against different types of pests.

\section{Antifungal Activity:-}

Antifungal activity was carried out according to food poisoning method (Siressha.O 2013) on fungus Sclerotinia sclerotiorum (SS). Sclerotinia sclerotiorum is a plant pathogenic fungus and can cause a disease called white mold. It infests wide range of vegetables. Potato dextrose Agar (PDA) was used as media. The dose was added into media. Fungus was allowed to grow on media in BOD at $\mathbf{2 5}^{\mathbf{0}} \mathbf{C}$. The fungus growth was observed after $48 \mathrm{~h}, 72 \mathrm{~h} \& 96 \mathrm{~h}$. After each time interval, fungus growth was recorded as radius of fungus growth, which is further compared with blank and control. The results are given in table 4

\section{Nematicidal Activity Test:-}

In vitro Nematicidal activity test was carried out on sample S1, S2 and S3. The root knot nematode Meloidogyne graminicola was tested on different sample concentrations. 500ul of nematode suspension containing $10-15$ nematodes count was treated with $500 \mathrm{ul}$ of sample concentration. Mortality was observed after $24 \mathrm{~h}, 48 \mathrm{~h}$ and $96 \mathrm{~h}$. (Table 5 shows Nematodes count perml and No. of dead Nematodes at different time interval). \% mortality is shown graphically in Graph 1.

\section{Antifeedancy Test:-}

Antifeedancy test was carried according leaf Disc no choice method (Jaipal Singh Choudhary 2014). The third instar larvae of Spodoptera litura was tested insect. It was allowed to feed on castor leaf. Third instar larvae of insects was starved for $24 \mathrm{hrs}$. Castor leaf was properly washed with water and cut into uniform shape of equal diameter. $2 \%$ solution of WDG dispersed in distilled water was used. The different concentrations were sprayed on the leaves and 
allow it to dry for 20s. Prestarved larvae were allowed to feed on treated leaf for $24 \mathrm{~h}$ and $48 \mathrm{~h}$. Unconsumed area of leaf disc was recorded with leaf area meter. Larval mortality and pupal deformities was also recorded. The results are given in table 6

\section{Results and Discussion:-}

\section{Suspensibility, Dispersibility and Particle size:-}

The quality parameters Suspensibility, Dispersibility and Particle Size of three samples showed no remarkable difference when compared with each other. Sample S1 has highest suspensibility of 85\% and Sample S3 has lowest Suspensibility $79 \%$. Dispersibility of S1 is highest $88 \%$ and S3 has lowest $82 \%$. The particle size corresponds to suspensibility. Higher the suspensibility lower is the particle size.

Table 3:- Suspensibility, Dispersibility and Particle Size of Samples

\begin{tabular}{|l|l|l|l|}
\hline Sample Name & Suspensibility(\%) & Dispersibility(\%) & Particle $\operatorname{size}(\mathbf{m i c r o n s})$ \\
\hline S1 & 85 & 88 & 12 \\
\hline S2 & 82 & 86 & 10 \\
\hline S3 & 79 & 82 & 15 \\
\hline
\end{tabular}

\section{Antifungal Activity:-}

In sample S1 \& S2 after 48hours growth has started, whereas no growth was observed in S3 till $96 \mathrm{~h}$. The blank at $48 \mathrm{~h}$ has fungus growth of radius $4.1 \mathrm{Cm}$ which further reached to $8 \mathrm{~cm}$ after $96 \mathrm{~h}$. Whereas in sampleS1 after $96 \mathrm{~h}$ has $4.2 \mathrm{~cm}$ radius of fungus extended, $4.0 \mathrm{~cm}$ in $\mathrm{S} 2$ and no growth in $\mathrm{S} 3$ compared to blank $(8 \mathrm{~cm}) . \mathrm{S} 1$ \& $\mathrm{S} 2$ were showing almost same effect with negligible change. S3 can be considered best sample it showed no growth till $48 \mathrm{~h}$ and even after $96 \mathrm{~h}$ it has no fungus growth. It can be because Sample S3 has mixture of nanoparticles suspension (Cu+Ag) which together suppressing fungal growth. Copper and silver nanoparticle both have their own antifungal properties as shown in S1\&S2.when copper and silver Nanoparticle used in combination they show potential to suppress fungal growth.

Table 4:- Antifungal activity in different samples

\begin{tabular}{|c|c|c|c|c|}
\hline Time (hrs) & Blank & Sample(S1) & Sample(S2) & Sample(S3) \\
\hline $\mathbf{4 8}$ & 4.1 & Growth started & Growth started & No Growth \\
\hline $\mathbf{7 2}$ & 6.3 & 3.4 & 3.2 & Growth Started \\
\hline $\mathbf{9 6}$ & 8.0 & 4.2 & 4.0 & 0 \\
\hline
\end{tabular}

\section{Nematicidal Activity:-}

Nematicidal Activity of S3 was highest in 24h, 48h \& 96h, Sample S1 has more mortality\% as compared to S2 in $24 \mathrm{~h}, 48 \mathrm{~h} \& 96 \mathrm{~h}$, whereas in blank the mortality $\%$ was only $10 \%$ in $96 \mathrm{~h}$ and there was no mortality at $24 \mathrm{~h}$ and $48 \mathrm{~h}$. The mortality in one nematode may be because of infirmity, so death of one single Nematode is considered negligible out of 10. Same trend is observed here also as was in case of antifungal activity here is also S3 has maximum mortality of $73 \%$ after $96 \mathrm{~h}$, the effect may be again attributed to mixture of nanoparticles used in combination in formulation which together enhancing the property. 
Graph 1:- Showing \% Mortality in Nematodes at different Time Intervals)

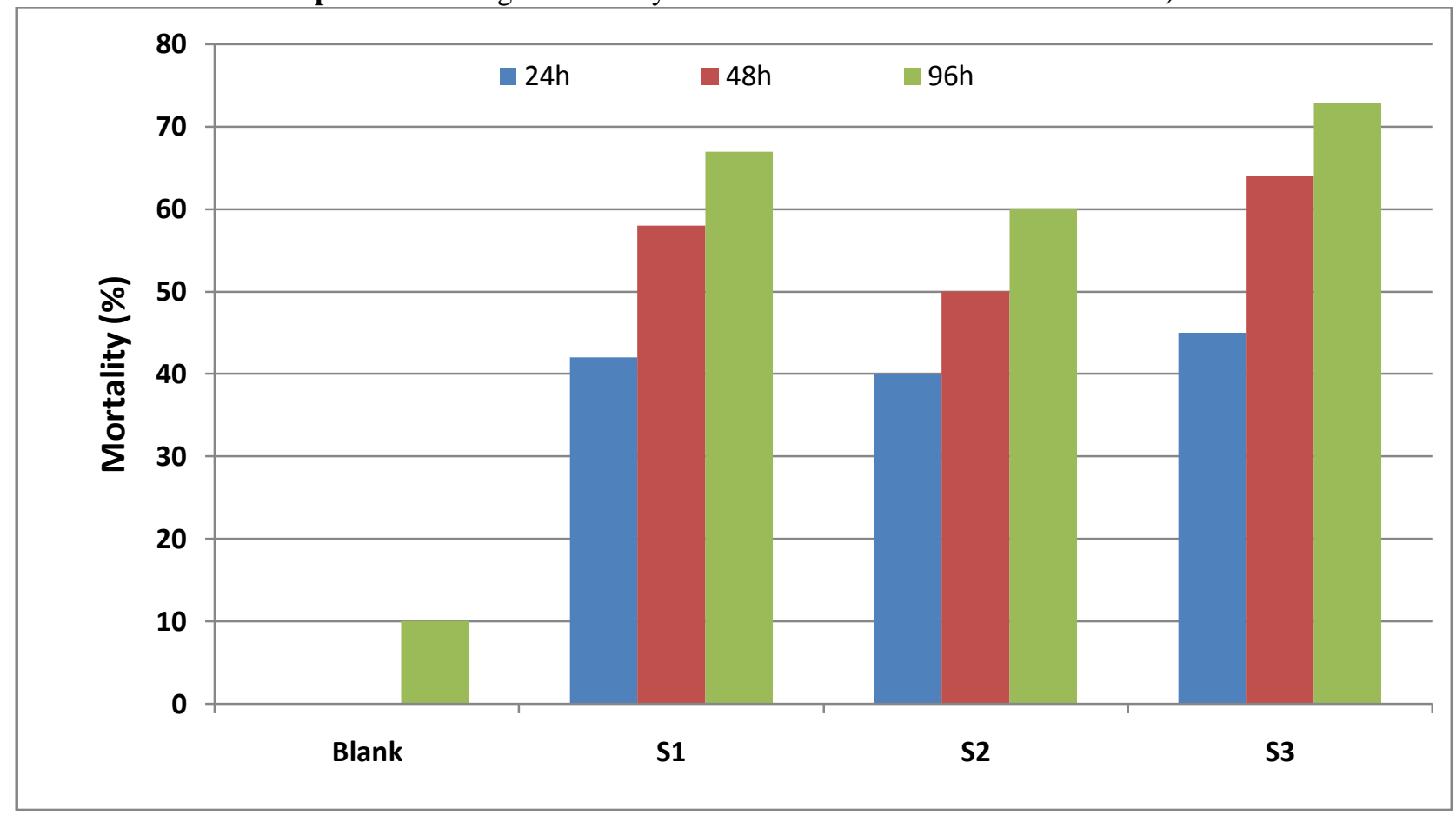

\section{Antifeedancy Test:-}

Antifeedancy of S1 in 24h and 48h were 20\% and 35\% respectively and in S2 20\% and 39\% respectively and in S3 $33 \%$ and $42 \%$ respectively, whereas in blank only $10 \%$ and $16 \%$ mortality was observed in $24 \mathrm{~h}$ and $48 \mathrm{~h}$ respectively. There is no significant diference in antifeedancy\% in all three samples. All samples following almost same trend. Antifeedancy varies in $39 \pm 5$ in $48 \mathrm{~h}$ and in $24 \mathrm{~h} \mathrm{S3}$ shows highest antifeedancy as compared to S1 and S2 with a difference of $(33-20=13 \%)$

Table 6:- Antifeedancy \% of Samples

\begin{tabular}{|l|c|c|}
\hline Sample & \% Antifeedancy (24h) & \% Antifeedancy (48h) \\
\hline S1 & 20 & 35 \\
\hline S2 & 20 & 39 \\
\hline S3 & 33 & 42 \\
\hline Blank & 10 & 16 \\
\hline
\end{tabular}

\section{Conclusion:-}

All three samples have negligible changes in quality parameters suspensibility, Dispersibility and particle size. Suspensibility corresponds to particle size in all samples. As far as efficacy part is concerned all three samples have shown remarkable effect in antifungal activity and Nemeticidal activity in comparision of blank. The antifeedancy of samples as compared to blank was not much significant. However when three samples compared to each other S3 was best among other two samples. It shows significant antifungal activity and has no fungal growth even after $48 \mathrm{~h}$ and only $2.0 \mathrm{~cm}$ growth after $96 \mathrm{~h}$. S3 also show upto $73 \%$ mortality in nematodes population after $96 \mathrm{~h}$ which was highest among all samples, also S3 provides heighest $42 \%$ antifeedancy after 48 hrs. Since S3 contains mxture of two nanoparitcles $(\mathrm{Cu}+\mathrm{Ag})$ the two together attributes good performance in all activity. The prepared formulation shows antifungal, nematicidal and antifeedant properties so it can be said that there are multiple activities in single formulation. 


\section{Refrences:-}

1. H.J. Niessen, H. Hausmann, H. Neumaier(1983) dispersibility and friability of water-dispersible granules, Pesticide Chemistry: Human Welfare and the Environment 317-322

2. Knowles, A. (2008), Recent developments of safer formulations of agrochemicals. Environmentalist, 28,35-44

3. D. Ian Wilson, Sarah L. Rough Chapter 3 Extrusion-Spheronisation, Handbook of Powder Technology, Volume 11, 2007, Pages 189-217,

4. CIPAC Handbook, (2007) Physico-chemical methods for technical and formulated pesticides. Vol. F. Collaborative International Pesticide Analytical Council, Harpenden, U.K.

5. CIFAP(1989) Catalogue of pesticide formulation types and international coding system. Technical monograph no. 2. International group of national associations of manufacturers of agrochemical products , Brussels

6. International Journal of Engineering Research \& Technology (IJERT), Vol. 2 Issue 3, March - 2013, Synthesis of Silver Nanoparticles by using Sodium Borohydride as a Reducing Agent, Kandarp Mavani and Mihir Shah.

7. Trans. Nonferrous Met. Soc. China 22(2012) 2198-2203, Preparation of Cu nanoparticles with ascorbic acid by aqueous solution reduction method ,LIU Qing-ming, Takehiro YASUNAMI ,Kensuke KURUDA, Masazumi OKIDO.

8. Research Journal of Microbiology, 9: 34-42, Antifungal Activity of Silver and Copper Nanoparticles on Two Plant Pathogens, Alternaria alternata and Botrytis cinerea, Sahar M. Ouda, 2014.

9. Bioscience, Biotechnology, and Biochemistry, Several Antifeedants from Neem Oil, Azadirachta indica A. Juss., against Reticulitermes speratus Kolbe (Isoptera: Rhinotermitidae), Munetaka Ishida, Muney Serit, Katsuyasu Nakata, Lekh Raj Juneja, Mujo Kim \& Shozo Takahashi 1992.

10. The Bioscan: screening for antifeedant activity of gymnema sylvestre leaf extracts against spodoptera litura $\mathrm{f}$. (lepidoptera: noctuidae). jaipal singh choudhary, chitra srivastava, and suresh walia, 9(2): 633-638, 2014.

11. Int.J.Curr.Microbiol.App.Sci, Antifungal Activity of leaf extract of Neem (Azadirachta Indica Linn), D.K.Shrivastava and Kshma Swarnkar, 2319-7706 Volume 3 Number 5 (2014) pp. 305-308.

12. Asian Journal of Phrmaceutical and clinical Research, in vitro evaluation of botanicals and panchagavya against leaf blast fungus pyricularia grisea, sireesha.o venkateswarlu. n, Vol 6, Suppl 5, 2013

13. Lee SE, Kim JE, Lee HS (2001) Insecticide resistance in increasing interest. Agric Chem Biotechnol 44:105112

14. Macedo ME, Consoli RAGB, Grandi TSM, Anjos AMG, Oliveira AB, Mendes NM, Queiroz RO, Zani CL (1997) Screening of Asteraceae (Compositae) plant extracts for larvicidal activity against Aedes_fluvilatilis (Diptera: Culicidae). MemInstOswaldoCruz92:565-570

15. Trans. Nonferrous Met. Soc. China 22(2012) 2198-2203 LIU Qing-ming1, 2, Takehiro YASUNAMI 1, Kensuke KURUDA 1, Masazumi OKIDO, Preparation of $\mathrm{Cu}$ nanoparticles with ascorbic acid by aqueous solution reduction method 